\title{
A novel cell-penetrating peptide protects against neuron apoptosis after cerebral ischemia by inhibiting the nuclear translocation of annexin A1
}

\author{
Xing $\mathrm{Li}^{1,2,3} \cdot$ Lu Zheng ${ }^{1,2,3} \cdot$ Qian Xia ${ }^{1,2,3} \cdot{\text { Lu Liu } \mathbb{1}^{1,2,3} \cdot \text { Meng Mao }}^{1,2,3} \cdot$ Huijuan Zhou ${ }^{1,2,3} \cdot$ Yin Zhao $^{4} \cdot$ Jing Shi ${ }^{1,2,3}$
}

Received: 30 December 2017 / Revised: 28 March 2018 / Accepted: 5 April 2018 / Published online: 16 May 2018

(c) ADMC Associazione Differenziamento e Morte Cellulare 2018

\begin{abstract}
Nuclear translocation of annexin A1 (ANXA1) has recently been reported to participate in neuronal apoptosis after cerebral ischemia. Prevention of the nuclear translocation of ANXA1 should therefore inhibit neuronal apoptosis and protect against cerebral stroke. Here, we found that, in the repeat III domain of ANXA1, the amino-acid residues from R228 to F237 function as a unique nuclear translocation signal (NTS) and are required for nuclear translocation of ANXA1. Intriguingly, we synthesized a cell-penetrating peptide derived by conjugating the trans-activator of transcription (Tat) domain to the NTS sequence. This Tat-NTS peptide specifically blocked the interaction of ANXA1 with importin $\beta$ and, consequently, the nuclear translocation of ANXA1 without affecting the nucleocytoplasmic shuttling of other proteins. The Tat-NTS peptide inhibited the transcriptional activity of p53, decreased Bid expression, suppressed activation of the caspase- 3 apoptosis pathway and improved the survival of hippocampal neurons subjected to oxygen-glucose deprivation and reperfusion in vitro. Moreover, using a focal brain ischemia animal model, we showed that the Tat-NTS peptide could be efficiently infused into the ischemic hippocampus and cortex by unilateral intracerebroventricular injection. Injection of the Tat-NTS peptide alleviated neuronal apoptosis in the ischemic zone. Importantly, further work revealed that administration of the TatNTS peptide resulted in a dramatic reduction in infarct volume and that this was correlated with a parallel improvement in neurological function after reperfusion. Interestingly, the effects of Tat-NTS were injury specific, with little impact on neuronal apoptosis or cognitive function in sham-treated nonischemic animals. In conclusion, based on its profound neuroprotective and cognitive-preserving effects, it is suggested that the Tat-NTS peptide represents a novel and potentially promising new therapeutic candidate for the treatment of ischemic stroke.
\end{abstract}

\section{Edited by M. Piacentini.}

Electronic supplementary material The online version of this article (https://doi.org/10.1038/s41418-018-0116-5) contains supplementary material, which is available to authorized users.

Jing Shi

sj@mails.tjmu.edu.cn

1 Department of Neurobiology, Tongji Medical College, Huazhong University of Science and Technology, Wuhan 430030 Hubei Province, China

2 Key Laboratory of Neurological Diseases, Ministry of Education, Wuhan 430030 Hubei Province, China

3 The Institute for Brain Research, Collaborative Innovation Center for Brain Science, Huazhong University of Science and Technology, Wuhan 430030 Hubei Province, China

4 Department of Ophthalmology, Tongji Hospital, Tongji Medical College, Huazhong University of Science and Technology, Wuhan 430030 Hubei Province, China

\section{Introduction}

Cerebral ischemia is one of the leading causes of death and is correlated with high incidences of disability and morbidity worldwide [1, 2]. However, limited advances have been achieved in developing treatments to counter the deleterious effects of cerebral ischemia [3, 4]; currently, the only available therapy is thrombolysis by administration of tissue plasminogen activator (tPA) within $3-4.5 \mathrm{~h}$ of stroke onset $[5,6]$. It is widely accepted that neuronal apoptosis is the primary factor involved in the pathophysiology of cerebral ischemia [7, 8]. Thus, targeted inhibition of proapoptotic factors would provide a beneficial therapeutic strategy for ischemic stroke.

Annexin A1 (ANXA1) is a $37-\mathrm{kDa}$ member of the annexin superfamily of proteins [9]. Since its discovery, ANXA1 has been reported to participate in diverse cellular processes [10-12]. There is also overwhelming evidence for 

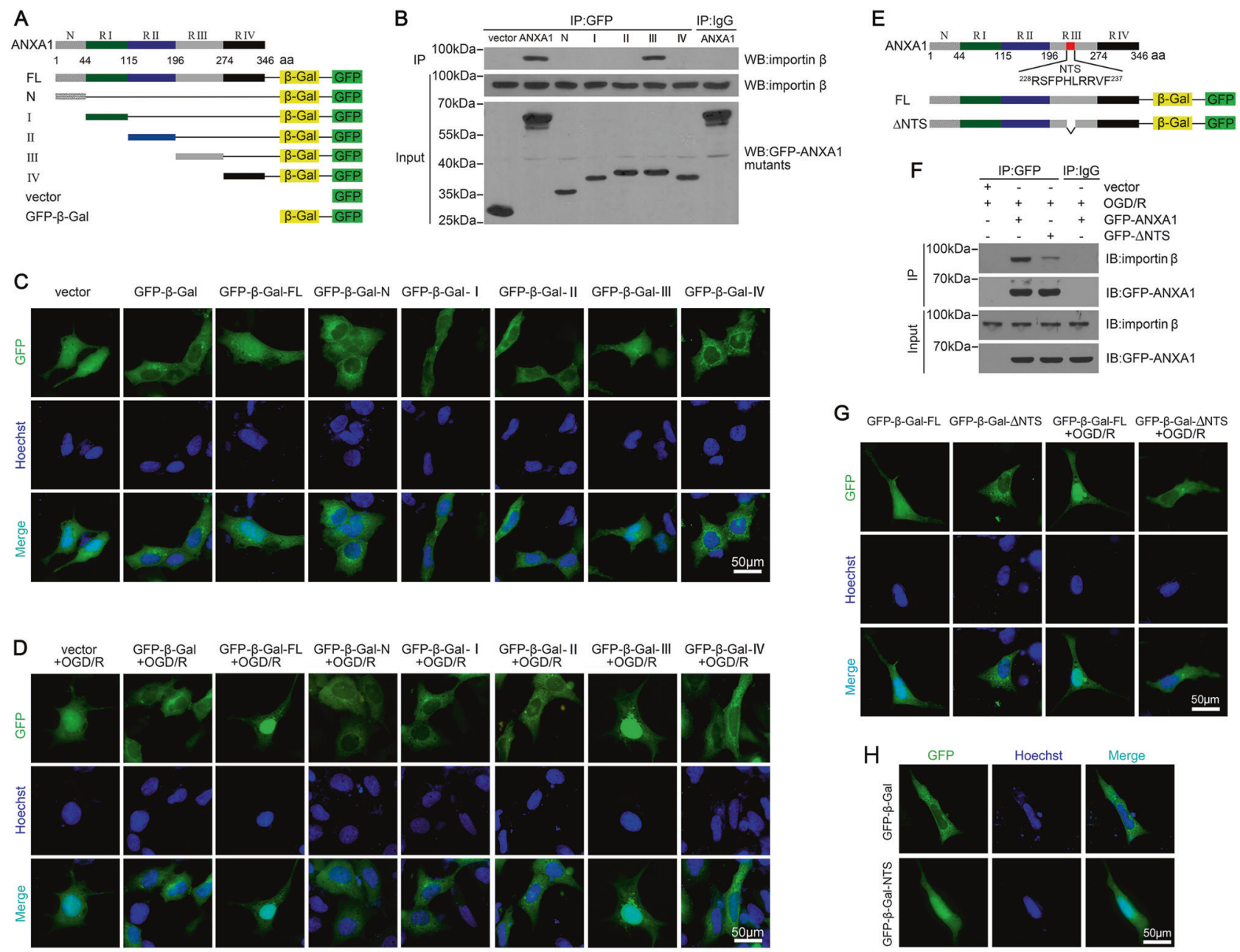

Fig. 1 Nuclear translocation of ANXA1 is mediated by amino-acid residues R228-F237 in the C-terminal core domain. a Schematic representation of the GFP- $\beta$-Gal tagged ANXA1 mutant constructs. The core domain is shown as four boxes at the carboxyl terminus, with each box depicting a repeat structure. b Representative Co-IP results show the interactions of full-length ANXA1 and various mutant constructs with importin $\beta$ in HEK293 cells treated with oxygenglucose deprivation and reperfusion (OGD/R). c, d GFP- $\beta$-Gal tagged full-length (FL) and various mutant constructs of ANXA1 were transfected into HEK293 cells and visualized by fluorescence microscopy. e Schematic of full-length ANXA1 and deletion NTS ( $\Delta$ NTS)

a role of intracellular ANXA1 in neuronal apoptosis following ischemic stroke [13-15]. In a previous study, we showed that importin $\beta$-dependent nuclear ANXA1 translocation is involved in oxygen-glucose deprivation and reperfusion (OGD/R)-dependent neuronal apoptosis [14]. As a co-factor, ANXA1 binds p53 in the nucleus and upregulates p53 transcriptional activity, which subsequently promotes pro-apoptotic Bid expression. Blocking the nuclear translocation of ANXA1 by importazole, a specific inhibitor of importin $\beta$, reduces the expression of Bid and inhibits activation of the caspase- 3 apoptotic pathway, consequently alleviating neuronal apoptosis after ischemic stroke [15]. However, the specific amino-acid residues that constructs used in this study. The red box represents the NTS aminoacid residues. NTS nuclear translocation signal. f Representative Co-IP results show the interactions of full-length ANXA1 and the $\triangle$ NTS mutant construct with importin $\beta$ in HEK293 cells. g GFP- $\beta$-Gal tagged full-length ANXA1 and the $\triangle$ NTS mutant construct of ANXA1 were transfected into HEK293 cells. Fusion proteins were visualized by fluorescence microscopy. h GFP tagged $\beta$-Gal and $\beta$-Gal-NTS were transfected into HEK293 cells and visualized by fluorescence microscopy. All data are representative of at least three independent experiments. Scale bar $=50 \mu \mathrm{m}$

functions as nuclear translocation signal (NTS) in ANXA1-importin $\beta$ interaction still need to be thoroughly addressed. On the other hand, because importazole inhibits importin $\beta$ [16], it not only inhibits the nuclear translocation of ANXA1 but also blocks the nucleocytoplasmic shuttling of other proteins $[17,18]$, and this may have an impact on the normal physiological function of the cell. Therefore, identification of an inhibitor that specifically blocks the nuclear translocation of ANXA1 might provide a promising target candidate for the treatment of cerebral stroke.

Trans-activator of transcription (Tat) is a cell-penetrating peptide and it is able to translocate numerous proteins, peptides, DNA, RNA, and small drugs into the cytoplasm in 

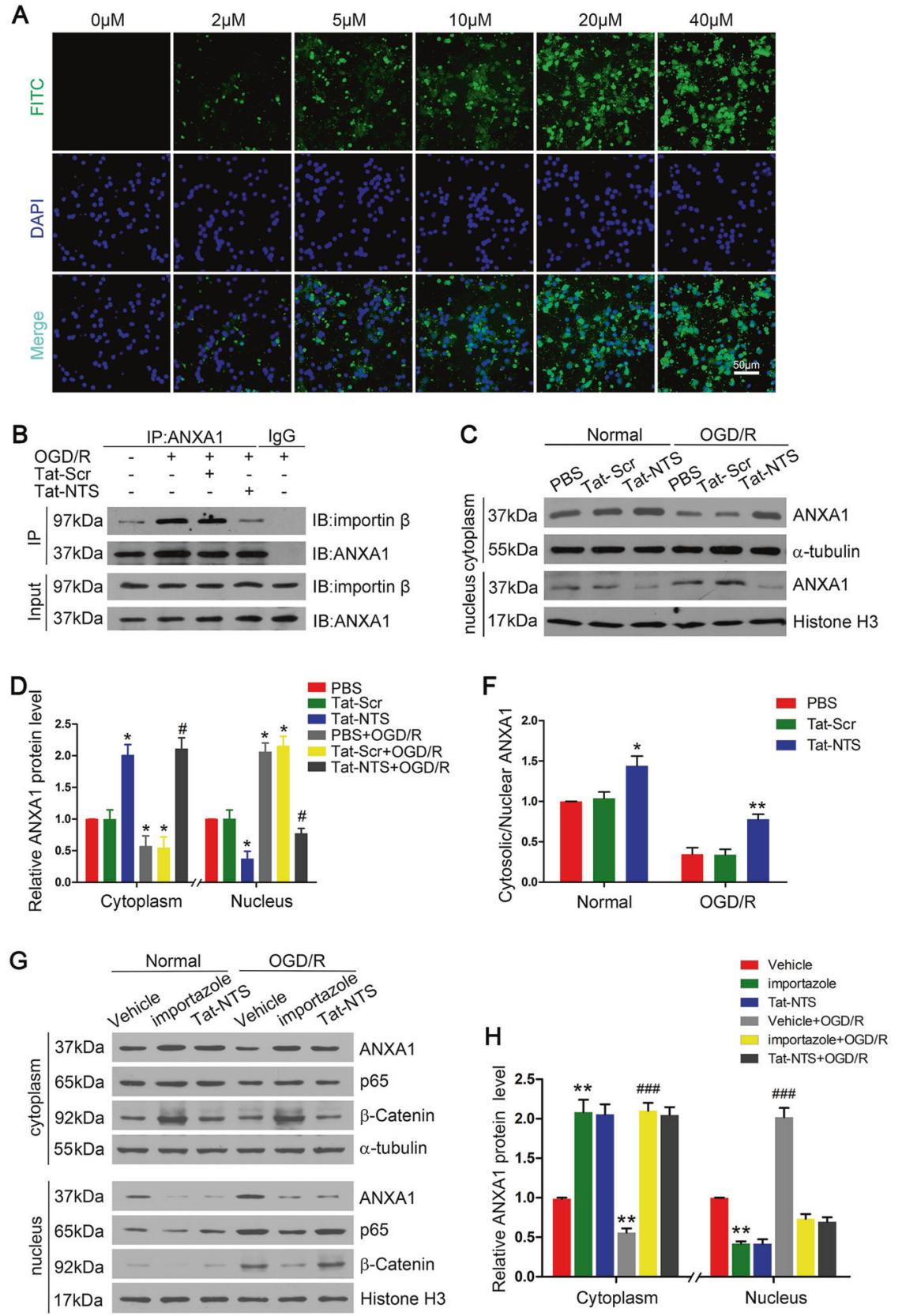
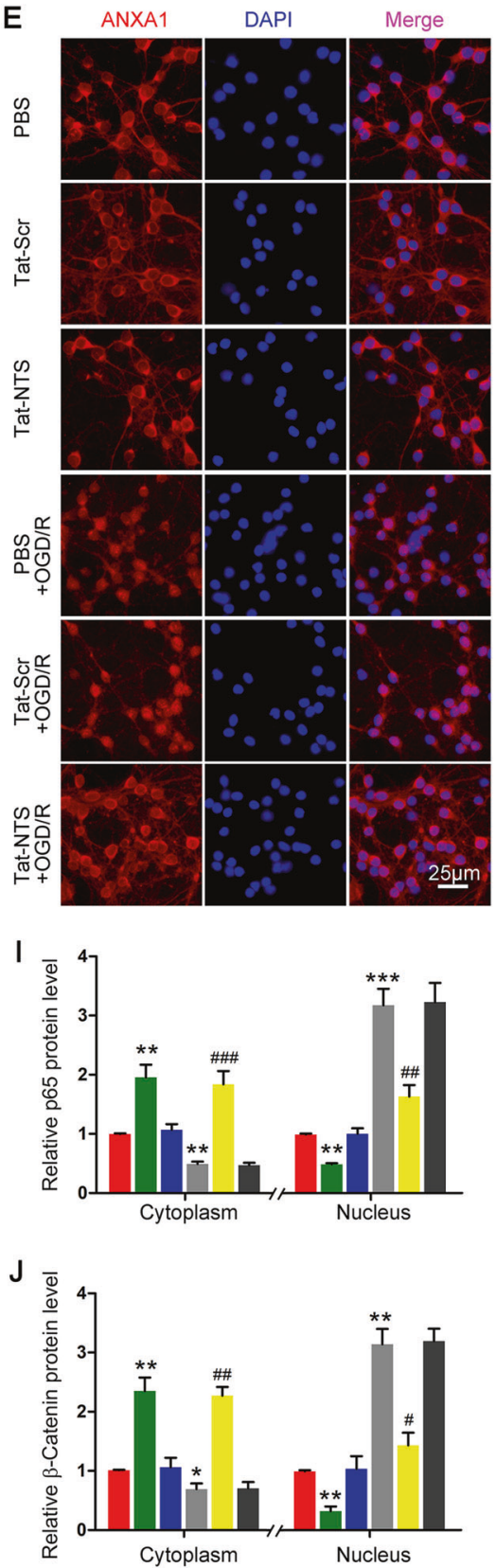

Fig. 2 Tat-NTS peptide blocks the interaction of ANXA1 with importin $\beta$ and specifically prevents nuclear translocation of ANXA1. a Dose-dependent penetration of FITC-Tat-NTS into primary cultured neurons. FITC was directly detected on not-fixed cells under fluorescence microscopy. Scale bar $=50 \mu \mathrm{m}$. b Representative Co-IP results showing the interaction of ANXA1 with importin $\beta$ in neurons treated with Tat-NTS or Tat-Scr peptide after exposure to OGD/R. c Western blotting analysis showing the protein level of ANXA1 in cytoplasmic and nuclear extracts of neurons that had been subjected to OGD/R. $\alpha$ Tubulin and Histone $\mathrm{H} 3$ were used as cytoplasmic and nuclear loading controls, respectively. d Quantification of the western blotting data shown in (c). The data are expressed as the mean \pm S.E.M. from three independent experiments. ${ }^{*} P<0.05$ vs. PBS; ${ }^{\#} P<0.05$ vs. PBS + OGD/R. e Immunofluorescence imaging of stained ANXA1 and DNA showing the subcellular distribution of endogenous ANXA1 in neuronal cells. Scale bar $=25 \mu \mathrm{m}$. f Quantitative analysis of the data shown in (e). The data are expressed as the mean \pm S.E.M. from three independent experiments. $* P<0.05$ vs. Tat-Scr; $* * P<0.01$ vs. $\mathrm{PBS}+\mathrm{OGD} / \mathrm{R}$. $g$ Immunoblots showing the relative amounts of ANXA1, NF- $\mathrm{KB}$ p65, and $\beta$-catenin in cytoplasmic and nuclear extracts of neurons treated with importazole, Tat-NTS peptide, or vehicle. $\alpha$-Tubulin and Histone H3 were used as cytoplasmic and nuclear loading controls, respectively. h, i, j Quantitative analysis of the protein levels of ANXA1, NF- $\mathrm{B}$ p 65 , and $\beta$-catenin. The intensity of the bands was quantitated by scanning densitometry and normalized to the values obtained for the control group. $* P<0.05$ vs. control; $* * P$ $<0.01 ; * * * P<0.001 ;{ }^{*} P<0.05$ vs. Vehicle $+\mathrm{OGD} / \mathrm{R} ;{ }^{\# \#} P<0.01$;

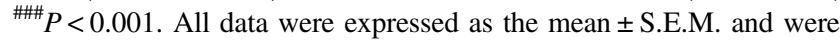
obtained in at least three independent experiments 
a short time with extremely high efficiency $[19,20]$. A large number of Tat fusion peptides and proteins have yielded promising results in various disease models and, in some cases, have been tested in clinical trials [21-24]. The outcome of these investigations has thus opened new perspectives for Tat application in the development of unprecedented human therapies. We hypothesized that the synthetic peptide Tat-NTS containing the NTS sequence of ANXA1 and the HIV-Tat cell transduction domain would specifically disrupt the interaction of ANXA1 with importin $\beta$ and thus inhibit the nuclear translocation of ANXA1, consequently protecting neurons from ischemic stroke damage.

In this study, we first investigated the amino-acid residues of ANXA1, which are critical for ANXA1-importin $\beta$ interaction and functions as a unique NTS. Next, we focused on whether the synthetic Tat-NTS peptide shows efficacy as a novel therapeutic candidate for ischemic stroke. We performed preclinical studies using an OGD/R cell model in vitro and the middle cerebral artery occlusion (MCAO) animal model of stroke in vivo to test this hypothesis. The results show here for the first time that treatment with Tat-NTS peptide results in robust neuroprotection, demonstrating that the Tat-NTS peptide may be suitable for development as a potential therapeutic agent in cerebral ischemic-reperfusion injury.

\section{Results}

\section{Identification of key NTS amino-acid residues for the nuclear translocation of ANXA1}

To identify which ANXA1 domains were required for nuclear translocation, mutations were made in five regions according to the structure of ANXA1 protein [25, 26]; the $\mathrm{N}$-terminal domain and four repeat domains in the Cterminal: repeats I, II, III, and IV (Fig. 1a). For fluorescence assay, as proteins of $30-40 \mathrm{kDa}$ or less in size can diffuse passively into the nucleus, so all mutation constructs of ANXA1 were fused to green fluorescent protein (GFP, 26 $\mathrm{kDa})$ with $\beta$-galactosidase ( $\beta$-gal, $112 \mathrm{kDa})$ [27]. Coimmunoprecipitation (Co-IP) results showed that only the full-length ANXA1 and repeat III domain could bind with importin $\beta$ (Fig. 1b). Immunofluorescence analysis confirmed that the full-length ANXA1 and repeat III domain were mostly localized to the nucleus, whereas the other constructs remained restricted to the cytoplasm (Figs. 1c, d). These findings suggest a prominent role for the repeat III domain in the nuclear translocation of ANXA1.

To further delimit the NTS within the repeat III region, the sequence of the C-terminal repeat III domain was evaluated for the presence of classical lysine or arginine-rich basic residues $[28,29]$. The crystal structure of ANXA1 had been solved and indicated that the repeat III domain consisted of four $\alpha$-helices and three loop structures [26]. Sequence analysis of each $\alpha$-helices and loop structures revealed that residues $228-237$ of the third $\alpha$-helix included these basic residues (R228, R234, R235). Meanwhile, as the third $\alpha$-helix is accessible on the surface of ANXA1, it may be more available for recognition by importin $\beta$. Based on these above, we hypothesized that residues 228-237 might function as an unconventional NTS. To verify this notion, we produced full-length and 228-237 deletion ( $\Delta$ NTS) constructs of ANXA1. Figure 1e illustrates a schematic of this deletion. Co-IP results showed that the $\triangle$ NTS construct failed to bind with importin $\beta$ (Fig. 1f). Immunofluorescence analysis also showed that the $\Delta$ NTS construct distributed in the cytoplasm (Fig. 1g), suggesting that the Arg228-Phe237 residues could be a functional NTS of ANXA1. To exam whether the NTS are sufficient for nuclear translocation of a heterologous protein, we directly fused the NTS to the N-terminus of GFP- $\beta$-Gal fusion protein. Notably, GFP- $\beta$-Gal protein was mostly distributed in the cytoplasm, whereas a large part of fusion protein GFP- $\beta$-Gal-NTS localized predominantly in the nucleus suggesting that the NTS could direct the nuclear translocation of another cytosolic protein (Fig. 1h).

\section{Tat-NTS peptide specifically inhibits the nuclear translocation of ANXA1}

A cell-penetrating peptide derived by conjugating the Tat to the NTS sequence was synthesized. We first confirmed the membrane permeability of Tat-NTS peptide and established optimal conditions for its entry into primary cultured neurons. Fluorescein isothiocyanate-labeled Tat-NTS (FITCTat-NTS) entered neurons in a dose-dependent manner; the best results were obtained at concentrations of $20-40 \mu \mathrm{M}$ (Fig. 2a). Therefore, we used a 20- $\mu \mathrm{M}$ dose of the peptide for all of the in vitro experiments in this study. We then conducted Co-IP experiments to determine the efficacy of Tat-NTS in blocking ANXA1-importin $\beta$ interaction. As shown in Figure 2b, Tat-NTS treatment markedly decreased ANXA1 interaction with importin $\beta$. Furthermore, Tat-NTS peptide robustly decreased ANXA1 migration to the nucleus, whereas the Tat-Scr peptide had little effect on the induction of nuclear translocation of ANXA1 by OGD/R (Figs. 2c, d). These results were also validated by immunofluorescence staining analysis (Figs. 2e, f).

To determine the specificity of the effect of Tat-NTS peptide on ANXA1 translocation, we compared the effects of Tat-NTS peptide and importazole on the nuclear translocation of nuclear factor (NF)- $\mathrm{\kappa B}$ p65, $\beta$-catenin and ANXA1. NF- $\kappa \mathrm{B}$ p65 and $\beta$-catenin are nucleocytoplasmic shuttling proteins, and $\mathrm{OGD} / \mathrm{R}$ trigger their nuclear 
A

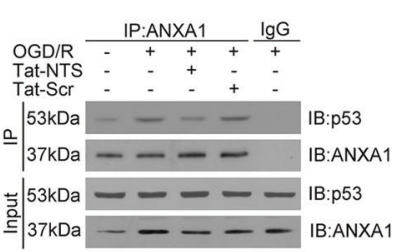

E

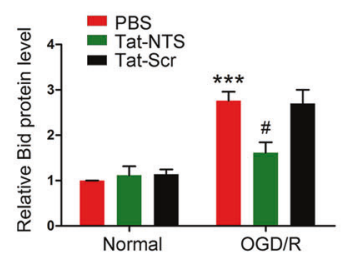

I

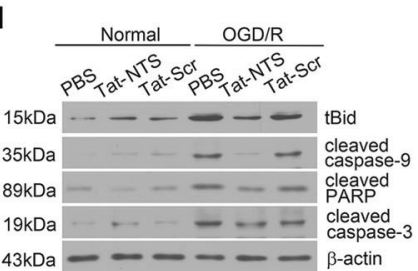

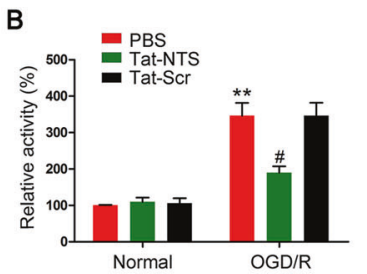

$\mathbf{F}$

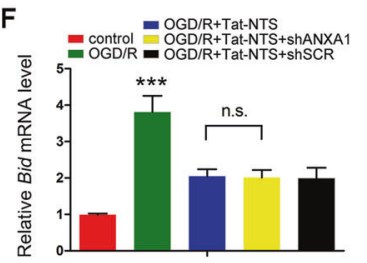

$J$

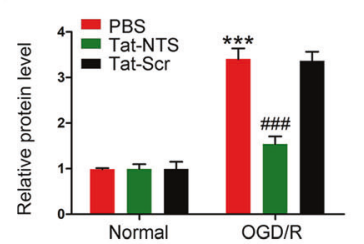

C

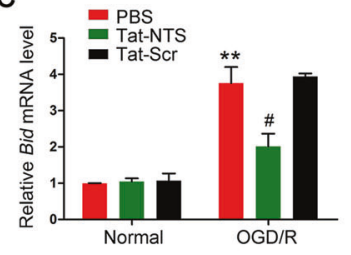

G

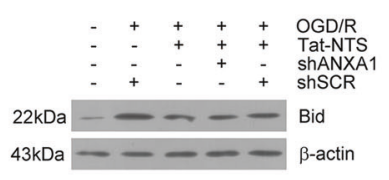

$\mathrm{K}$

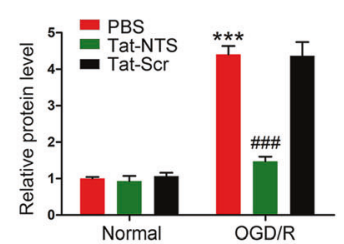

D

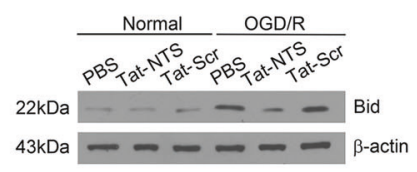

M

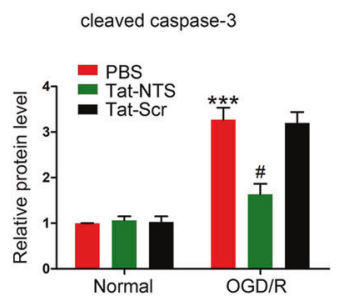

Q
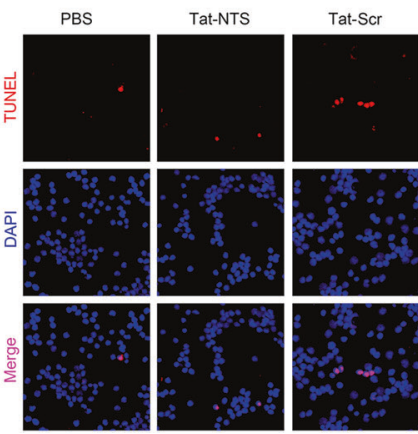

N

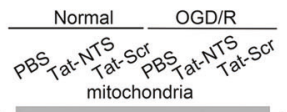

${ }_{14 \mathrm{kDa}}-0-1 \mathrm{~m}-\mathrm{Cytochrome} \mathrm{c}$

$17 \mathrm{kDa}---2 \mathrm{cox} I \mathrm{~V}$

cytoplasm

$14 \mathrm{kDa}$ - - - Cytochrome $c$

$43 \mathrm{kDa}---\beta$-actin
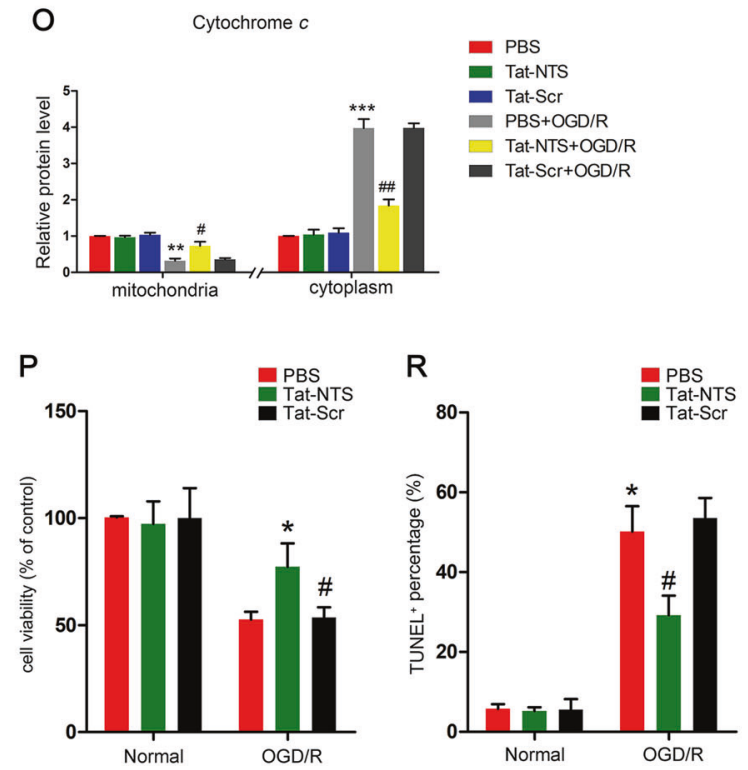

Fig. 3 Tat-NTS peptide treatment inhibits the binding of ANXA1 to p53 and attenuates OGD/R-induced neuronal apoptosis. a Representative Co-IP results showing the interaction of ANXA1 with p53 in neurons. $\mathbf{b}$ Luciferase assay results show the transcriptional activity of p53 in neuronal cells. c RT-qPCR analysis showing the effects of TatNTS peptide treatment on Bid mRNA expression in neurons subjected to OGD/R. d Immunoblots showing the Bid protein level in neurons subjected to OGD/R. e Statistical analysis of Bid protein expression. f Statistical analysis of the RT-qPCR results showing the expression of Bid mRNA after treatment of neurons with ANXA1 shRNA and TatNTS peptide. $\mathbf{g}$ Western blotting analysis showing the protein level of Bid after treatment with ANXA1 shRNA and Tat-NTS peptide. h Quantitative analysis of the data shown in (g). i Western blots showing the protein levels of tBid, cleaved caspase-9, cleaved PARP, and

cleaved caspase- 3 in primary cultured neurons. $\mathbf{j}-\mathbf{m}$ Statistical analysis of tBid, cleaved caspase-9, cleaved PARP, and cleaved caspase-3 expression. $\mathbf{n}$ Western blotting analysis showing the protein level of cytochrome $c$ in mitochondria and cytoplasm extracts of neurons. o Quantification of the protein level of cytochrome $c$. p OGD/R-induced changes in the viability of neuronal cells treated with Tat-NTS peptide measured using the CCK-8 assay. q, $\mathbf{r}$ Representative TUNEL staining and quantitative analysis showing the effect of Tat-NTS peptide treatment on neuronal apoptosis after OGD/R. Scale bar $=50 \mu \mathrm{m}$. In all graphs, the data are expressed as the mean \pm S.E.M. from three independent experiments. $* P<0.05$ vs. Normal control; $* * P<0.01$ vs. Normal control; $* * * P<0.001$ vs. Normal control; ${ }^{\#} P<0.05$ vs. PBS $+\mathrm{OGD} / \mathrm{R} ;{ }^{\# \#} P<0.01$ vs. $\mathrm{PBS}+\mathrm{OGD} / \mathrm{R},{ }^{\# \#} P<0.001$ vs. $\mathrm{PBS}+$ OGD/R. n.s., no significant difference between the two groups 
A

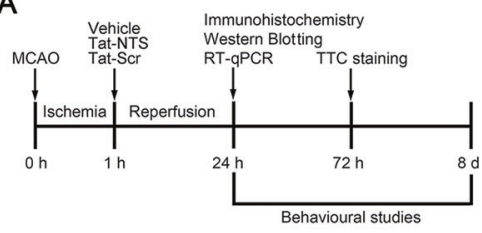

C

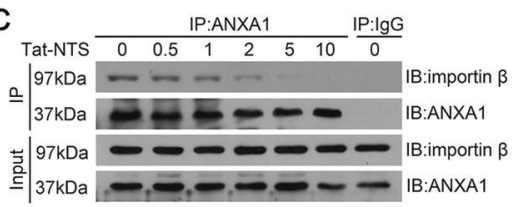

D

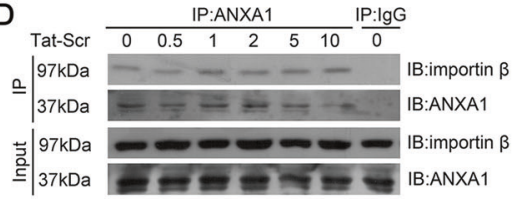

E

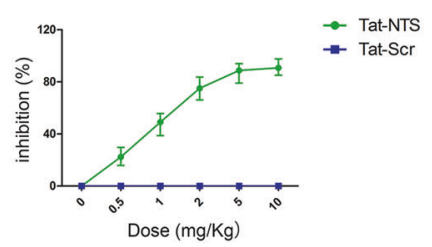

Fig. 4 The Tat-NTS peptide blocks the interaction of ANXA1 with importin $\beta$ and decreases the nuclear translocation of ANXA1 in mice. a Schematic diagram showing the experimental procedure. b Representative images showing the distribution of FITC labeled Tat-NTS peptide colocalized with nuclei in hippocampus and cortex of mouse brain. Scale bar $=50 \mu \mathrm{m}$. c, d Representative blots of Co-IP assays demonstrating the dose-response effects of Tat-NTS and Tat-Scr peptides on ANXA1-importin $\beta$ interaction. e Dose-response curves of Tat-NTS, and Tat-Scr peptides for inhibition of ANXA1-importin $\beta$ association in cortical cells in vivo. f Western blotting showing protein levels of ANXA1 in cytoplasmic and nuclear extracts. $\alpha$-Tubulin and
B

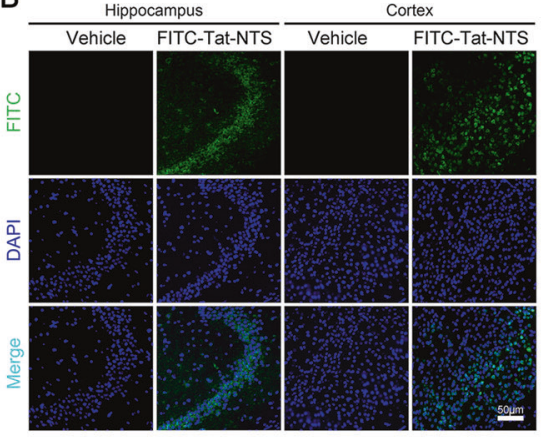

$\mathrm{F}$

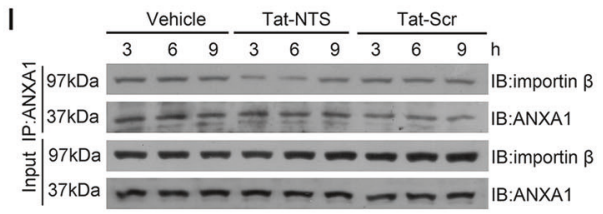

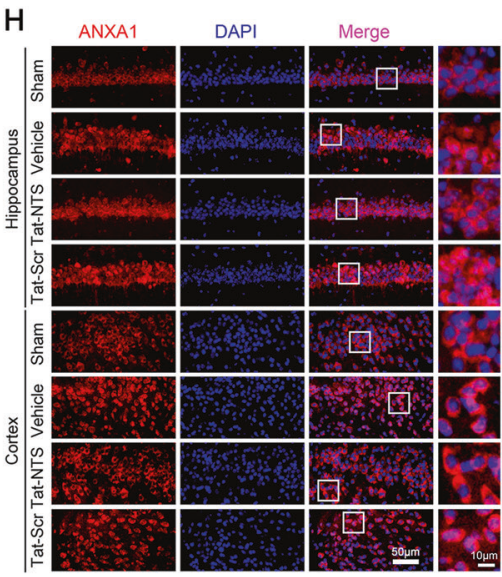

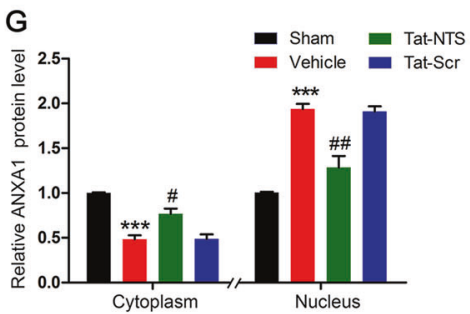

Histone H3 were used as cytoplasmic and nuclear loading controls, respectively. g Quantification of the western blotting data shown in (f). h Immunofluorescence imaging of stained ANXA1 and DNA showing the subcellular distribution of endogenous ANXA1 in hippocampus and cortex. Scale bar $=50$ or $10 \mu \mathrm{m}$ as indicated. i Representative blots of Co-IP assays demonstrating the time course of Tat-NTS peptide inhibition of ANXA1-importin $\beta$ interaction in cortical extracts. All the data are presented as the mean \pm S.E.M $(n=5$ mice per group). ${ }^{* * *} P<0.001$ vs. Sham; ${ }^{\#} P<0.05$ vs. Vehicle; ${ }^{\# \#} P<0.01$ vs. Vehicle

[15]. We therefore undertook to examine the effect of Tat-NTS on the interaction of ANXA1 with p53. Tat-NTS peptide decreased the interaction of ANXA1 with p53 triggered by OGD/R (Fig. 3a). The luciferase reporter assay results suggested that treatment with Tat-NTS peptide resulted in dramatically decreased p53 transcriptional activity (Fig. 3b). Next, reverse transcriptase-quantitative PCR (RTqPCR) and western blotting analysis suggested that Tat-NTS peptide significantly decreased Bid mRNA and protein levels, whereas the Tat-Scr peptide had no effect (Figs. 3c-e). To assess whether the inhibition of Bid expression by Tat-NTS peptide occurred specifically through the effect of ANXA1, an adenoviral vector encoding a short hairpin RNA (shRNA) against $A N X A 1$ was constructed. Transfection of neurons with the ANXA1 shRNA resulted in significant knockdown of ANXA1 expression (Supplementary Fig. 1). Consistent with previous reports, downregulation of ANXA1 resulted in a marked decrease in Bid expression. As shown in Figures 3f-h, compared with administration of Tat-NTS peptide alone, the 
A

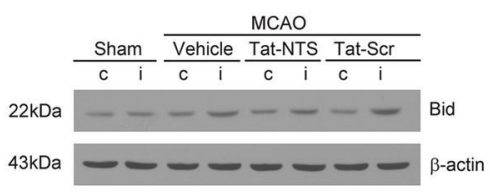

C

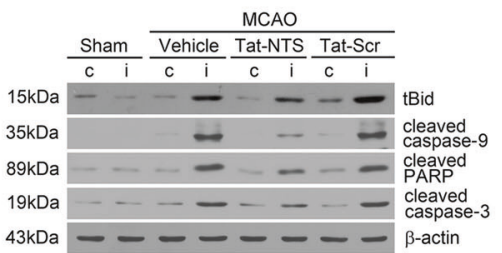

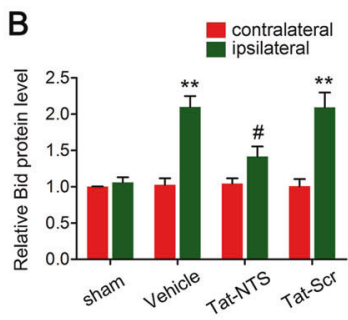

D

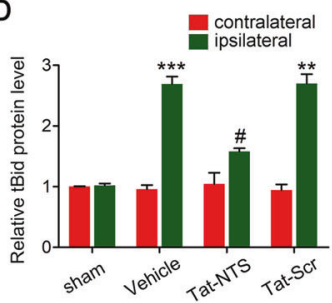

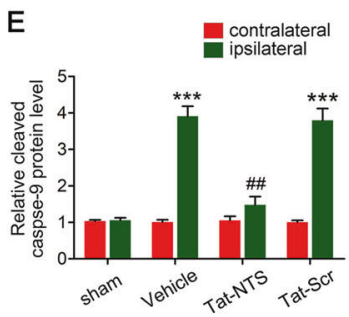

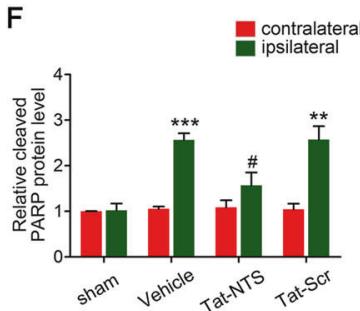

G

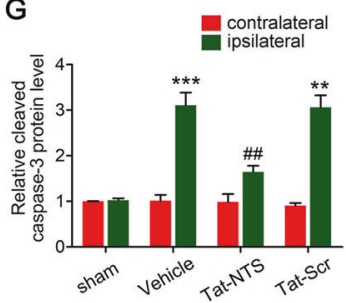

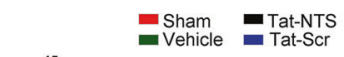

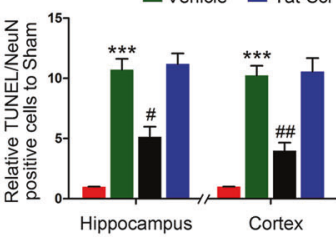

$\mathrm{H}$

Hippocampus
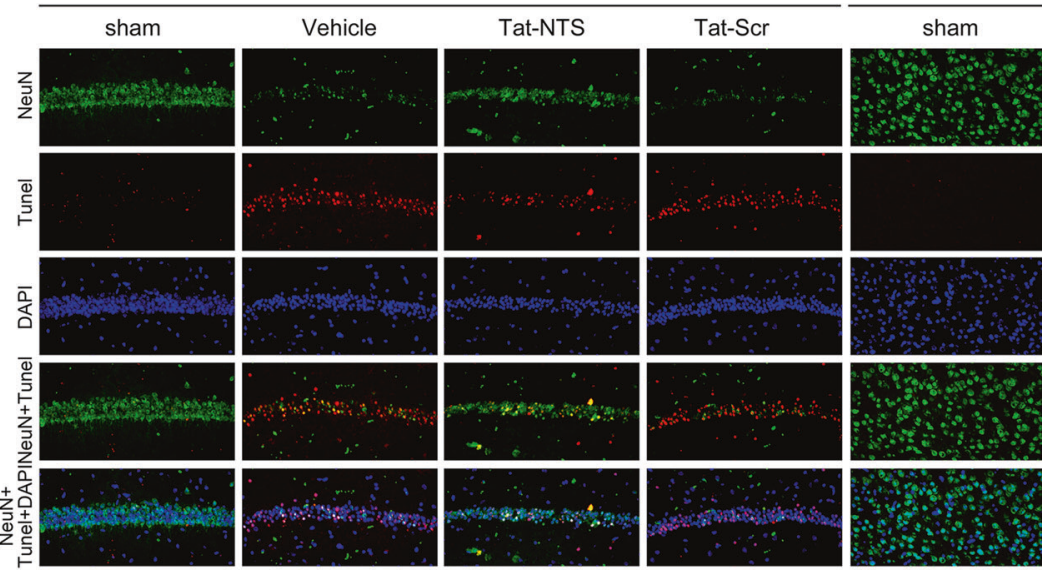

Cortex

Fig. 5 Tat-NTS peptide alleviates ischemia-induced neuronal apoptosis following ischemic injury in mice. a, b Bid protein level quantified by western blotting at $24 \mathrm{~h}$ of reperfusion after $1 \mathrm{~h}$ of MCAO (c contralateral hemisphere, $\mathrm{i}$ ischemic hemisphere). c Western blotting analysis of the expression of tBid, cleaved caspase-9, cleaved PARP, and cleaved caspase-3. d-g Quantification analysis of the protein levels of tBid, cleaved caspase- 9 , cleaved PARP, and cleaved caspase3 , respectively. h Representative hippocampal and cortical sections

from sham, Vehicle, Tat-NTS, and Tat-Scr-treated animals were subjected to double staining for NeuN and TUNEL. i Quantification was conducted by counting the number of NeuN-positive neurons and TUNEL-positive neurons. For all graphs, the data represent the mean \pm S.E.M. ( $n=5$ mice per group). $* * P<0.01$ vs. Sham; $* * * P<0.001$ vs. Sham; ${ }^{\#} P<0.05$ vs. Vehicle; ${ }^{\# \#} P<0.01$ vs. Vehicle. Scale bar $=$ $100 \mu \mathrm{m}$

expression of Bid was not further decreased in cells that were transfected with ANXAl shRNA and received Tat-NTS treatment.

It has been well documented that Bid needs to be cleaved by caspase- 8 or other proteases into truncated Bid (tBid) to become activated, thereby inducing the cytosolic release of cytochrome $c$ from the mitochondria after ischemia. In turn, the released cytochrome $c$ ultimately leads to the cleavage and activation of caspase- 9 and caspase- 3 , representing the final steps of the intrinsic caspase pathway that has been previously shown to regulate ischemic cell death $[34,35]$. To further confirm the protective effect of Tat-NTS peptide, the expression of tBid, cleaved caspase-9, cleaved PARP and cleaved caspase-3 was measured by western blotting. As shown in Figures 3i-m and Supplementary Figure 2, TatNTS treatment significantly decreased OGD/R-triggered tBid expression and caspase-9, PARP, caspase-3 cleavage but had little effect on the activation of caspase-8. Meanwhile, Tat-NTS markedly blocked the cytosolic release of cytochrome $c$ from the mitochondria (Figs. 3n, o). We next performed CCK- 8 assays to determine the viability of neurons. The results showed that administration of Tat-NTS peptide markedly increased cell viability after OGD/R (Fig. 3p). Moreover, TdT-mediated dUTP-X nick end labeling (TUNEL) staining revealed that OGD/R-triggered neuronal apoptosis was alleviated by treatment of the cells 
A
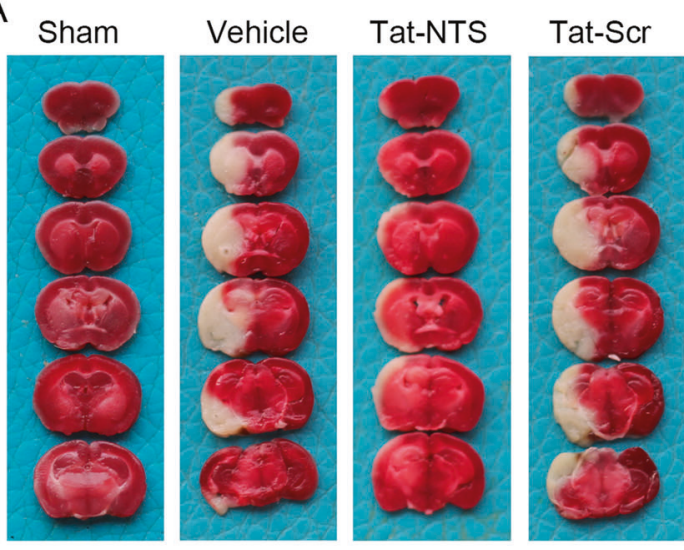

B
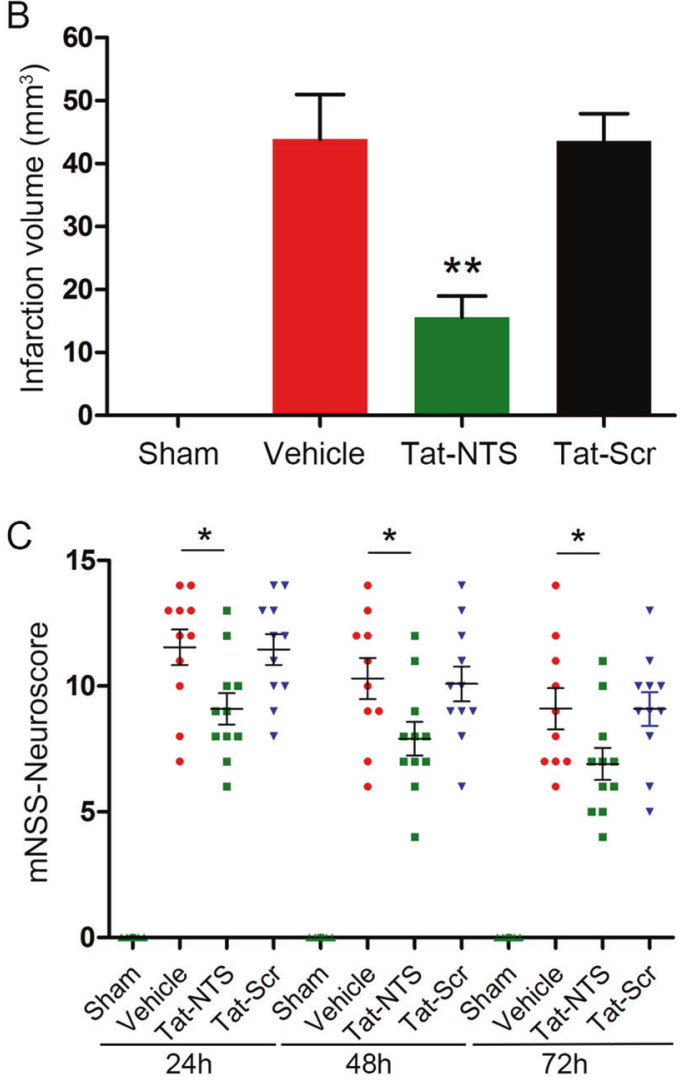

Fig. 6 Tat-NTS peptide treatment reduces infarct volume and decreases neurological deficit score following focal ischemic injury in mice. a Representative brain infarct size by TTC staining in mice treated with Vehicle, Tat-NTS, Tat-Scr peptide at $72 \mathrm{~h}$ after reperfusion. b Quantitative analysis of the rectified infarcted volume $\left(\mathrm{mm}^{3}\right)$ in each group. c The neurological outcomes were evaluated using the $\mathrm{mNSS}$ at 24, 48, and $72 \mathrm{~h}$ after reperfusion. All data are expressed as the mean \pm S.E.M. $(n=10-11$ mice per group). $* P<0.05$ vs. Vehicle, $* * P<0.01$ vs. Vehicle

with Tat-NTS peptide but not by the Tat-Scr peptide (Figs. 3q, r). Finally, our results showed that Tat-NTS did not decrease the viability or survival of hippocampal neurons under normal conditions, implying that it is safe and that it has no cytotoxic effects (Figs. 3i-r).
Tat-NTS peptide decreases interaction of ANXA1 with importin $\beta$ and blocks the nuclear translocation of ANXA1 in vivo

Adult C57BL/6 male mice were subjected to MCAO for 60 min followed by reperfusion. Cerebral ischemia was confirmed in all animals via monitoring cerebral blood flow; after reperfusion, behavioral and histological studies were performed at the indicated time points (Fig. 4a). As shown in Figure $4 b$, after unilateral intracerebroventricular (i.c.v.) injection of FITC-Tat-NTS, the peptide was efficiently infused into the cells and accumulated in cell bodies in the hippocampus and cerebral cortex regions. We then performed a dose-response analysis to determine the effective dose. After the induction of MCAO, animals were i.c.v. injected with Tat-NTS or Tat-Scr peptide at single doses of $0.5,1,2,5$, and $10 \mathrm{mg} / \mathrm{kg}$. We observed that Tat-NTS at a dose of $2 \mathrm{mg} / \mathrm{kg}$ sufficiently uncoupled ANXA1 protein from the importin $\beta$ complex. Therefore, a dose of $2 \mathrm{mg} / \mathrm{kg}$ was used in the remainder of the study (Figs. 4c-e). Administration of Tat-NTS peptide significantly reduced the level of ANXA1 protein in the nucleus (Figs. 4f, g). A similar decreasing trend in the level of ANXA1 protein was observed by immunofluorescence staining (Fig. 4h). To investigate the effective intervention time window, we administered Tat-NTS peptide $(2 \mathrm{mg} / \mathrm{kg}$, i.c.v. $)$ to mice at three time points (3, 6, and $9 \mathrm{~h}$ after reperfusion). Three and $6 \mathrm{~h}$ after reperfusion, Tat-NTS was effective in disrupting ANXA1-importin $\beta$ binding (Fig. 4i). Taken together, these data indicate that $2 \mathrm{mg} / \mathrm{kg}$ (i.c.v.) may be used as an effective dose and that administration of Tat-NTS peptide 6 $\mathrm{h}$ after reperfusion provides an appropriate treatment time window.

\section{Tat-NTS peptide alleviates ischemia-induced neuronal cell apoptosis following ischemic injury in mice}

We further tested whether administration of the Tat-NTS peptide to animals could inhibit the ischemia-induced neuronal cell apoptosis. The results revealed that Tat-NTS peptide-treated mice showed a marked decrease in Bid protein levels at $24 \mathrm{~h}$ after reperfusion (Figs. 5a, b). Moreover, the protein level of tBid, cleaved caspase-9, cleaved PARP, and caspase- 3 was significantly inhibited by TatNTS treatment (Figs. $5 \mathrm{c}-\mathrm{g}$ ). However, Tat-NTS had little effect on the activation of caspase-8 (Supplementary Fig. 3). We used TUNEL staining to examine the number of apoptotic cells in the hippocampus and cerebral cortex $24 \mathrm{~h}$ after reperfusion. As shown in Figures $5 \mathrm{~h}$ and I, 1-h MCAO followed by 24 -h reperfusion triggered a profound reduction in the number of surviving cells and an increase in the number of apoptotic cells in the hippocampus and cerebral 

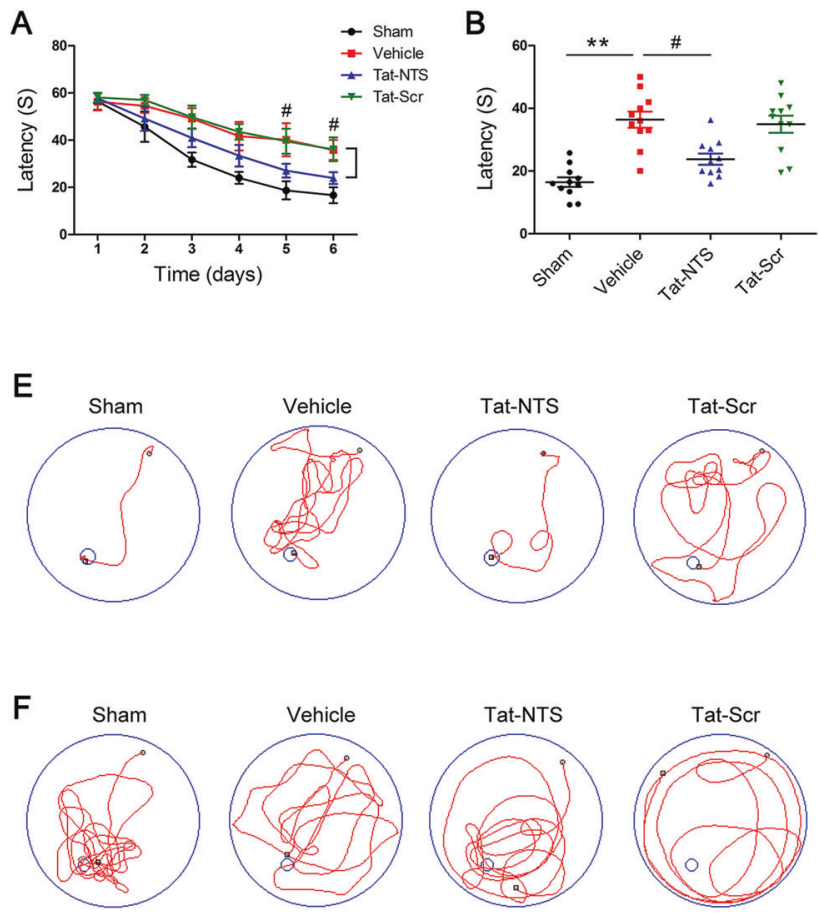

Fig. 7 Tat-NTS peptide treatment preserves cognitive and motor function following focal ischemic injury in mice. a-d Latency trial (a, b) and probe trial (c, d) results of sham treatment, MCAO with vehicle, Tat-NTS, and Tat-Scr peptide-treated mice in the MWM tests. a Escape latency to find the platform during days 1-6 after reperfusion of testing. b Exploration time spent in finding the submerged platform at 6 days following reperfusion during the latency trial. $\mathbf{c}$ Time spent in the safety quadrant during the probe trial. $\mathbf{d}$ Number of times the mice crossed over the platform location on day 6 (probe trial). e, f

cortex, whereas treatment with the Tat-NTS peptide was profoundly neuroprotective. These data collectively demonstrate that the Tat-NTS peptide improved neuronal survival and protected against cerebral ischemia-induced apoptosis.

\section{Tat-NTS peptide reduces infarct volume and improves neurological outcome following focal ischemic injury}

To further confirm the neuroprotective effects of Tat-NTS peptide against cerebral ischemia, we examined the infarct volume using 2,3,5-triphenyltetrazolium chloride (TTC) staining at $72 \mathrm{~h}$ after reperfusion. The results revealed that ischemic infarct volume was obviously reduced in Tat-NTS peptide-treated mice (Fig. 6a). Statistical analysis demonstrated that the rectified infarcted tissue volume was significantly decreased in Tat-NTS peptide-treated mice (Fig. 6b). Next, the neurological functional outcomes of mice were scored at three time points using the modified neurological severity score (mNSS). The mNSS neuroscores of Tat-NTS peptide-treated mice were consistently
C
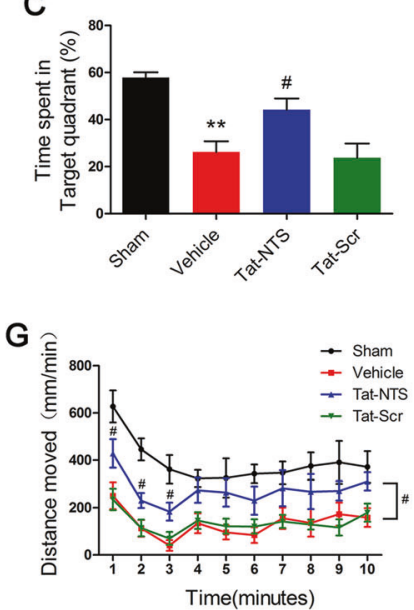

$\mathrm{H}$
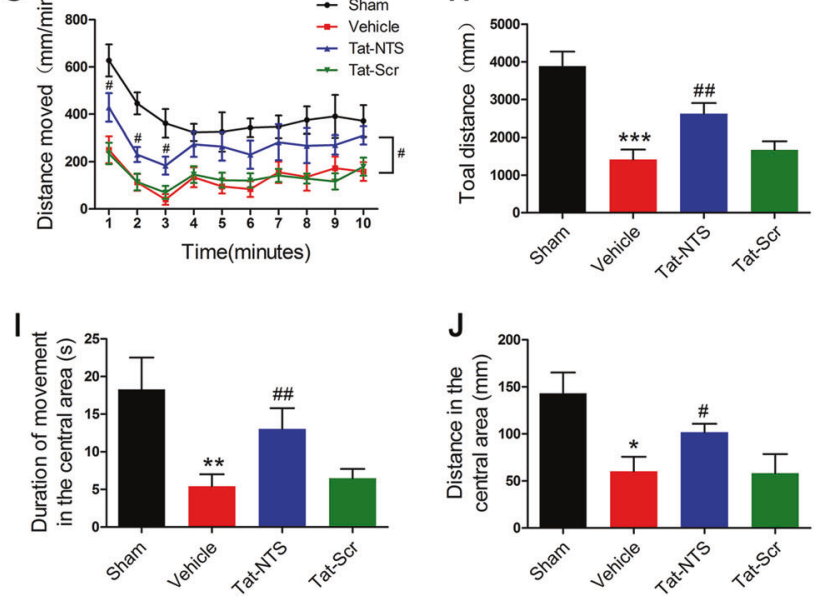

Representative traces indicating the sample paths of the mice from the maze latency trials (e), and the swimming traces from probe trials $\mathbf{f} . \mathbf{g}$-j Performance on the open-field test was analyzed throughout the 7-day period after MCAO in animals that were treated with vehicle, TatNTS, or Tat-Scr peptide. The data in all graphs are expressed as the mean \pm S.E.M. $(n=11$ mice per group). $* P<0.05$ vs. Sham; $* * P<$ 0.01 vs. Sham; $* * * P<0.001$ vs. Sham; ${ }^{\#} P<0.05$ vs. Vehicle; ${ }^{\# \#} P<$ 0.01 vs. Vehicle

markedly lower than those of vehicle- or Tat-Scr peptidetreated mice, indicating a significant correlation with the infarct size and reduced neurological deficit in Tat-NTS peptide-treated mice after cerebral ischemic injury (Fig. 6c).

\section{Tat-NTS peptide preserves neurobehavioral function following focal ischemic injury}

The Morris water maze (MWM) test was performed to ascertain spatial learning and memory function in mice, animals that underwent 1-h MCAO exhibited a significant increase in the time required to reach the hidden platform and a dramatic decrease in the time spent in the targeting quadrant compared with the sham control. However, TatNTS peptide-treated mice displayed remarkable cognitive improvement; the time required for the Tat-NTS peptidetreated animals to reach the hidden platform was considerably shorter than that required by the animals in the vehicle- and Tat-Scr-treated groups on the 5th and 6th days after injury (Figs. 7a, b). The Tat-NTS peptide-treated animals also exhibited a significant increase in the time spent in the targeting quadrant and crossed the platform 
location significantly more times on day 6 during the probe trials (Figs. 7c, d). Representative tracings indicating sample paths of the mice from the maze latency trials and swimming traces from probe trials are shown in Figs. 7e, f. We then used the open-field test to evaluate the animals' locomotion after surgery or treatment. The mice in the TatNTS group traversed a greater distance than those in the Tat-Scr group (Figs. $7 \mathrm{~g}, \mathrm{~h}$ ). Furthermore, the duration of movement in the central area was increased in the Tat-NTS group compared with the Tat-Scr group (Fig. 7i). The results suggest that the spontaneous locomotor activity of the model mice was improved by Tat-NTS peptide treatment (Fig. 7j).

To further assess the safety of treatment with Tat-NTS peptide, we examined the effect of Tat-NTS peptide on neuronal apoptosis and cognitive performance in shamtreated nonischemic mice. As shown in Supplementary Figures 4 and 5, the Tat-NTS peptide had no obvious effect on neuronal apoptosis, latency to find the platform, time spent in the targeting quadrant, or swim pattern. Taken together, these data collectively demonstrate that dissociation of ANXA1-importin $\beta$ interaction by administration of Tat-NTS peptide may offer a promising new therapeutic approach in ischemic stroke therapy.

\section{Discussion}

In the present study, we first identified the residues Arg228-Phe237 (RSFPHLRRVF) in the repeat III core domain of ANXA1 functions as an unconventional NTS. In light of this discovery, we synthesized a cell-penetrating peptide derived by conjugating the Tat domain to the NTS sequence. In particular, our results revealed that treatment with Tat-NTS peptide decreased ANXA1 nuclear translocation. Moreover, Tat-NTS peptide significantly alleviated cerebral ischemia injury-induced neuronal apoptosis. Furthermore, this study also showed that administration of the Tat-NTS peptide dramatically improved neurological functional outcome of mice subjected to brain ischemia injury.

Tat-mediated transduction has become a promising tool for the treatment of numerous diseases, including cerebral stroke [36]. A good example is Tat-NR2B9c, also termed as NA-1, which comprises of the last 9 amino acids of the carboxyl tail of GluN2B. This peptide binds to PSD95 with high specificity and perturbs synaptic GluN2B:PSD95: nNOS complex, thereby protecting neurons against NMDAR-mediated excitotoxicity. Tat-NR2B9c has been shown to safely and efficiently attenuate ischemic brain damage in cynomolgus macaques and human beings [21, 37-39]. In this study, the neuroprotective effect of the TatNTS was observed in the OGD/R cell model and the MCAO animal model of stroke. Therefore, the Tat-NTS peptide holds high potential for clinical application. As the Tat-NR2B9c and Tat-NTS peptide work through different mechanisms, we hypothesized that a combination of two different peptides would be even more potent. Certainly, more detailed work will be needed to verify the effect of this combination therapy. On the other hand, because Tat peptides enter various cell types with high efficiency in a nonselective manner [40], the effect of Tat-NTS treatment on astrocytes, oligodendrocytes, and microglia remains to be determined in future studies.

Cerebral ischemia causes significant damage resulting from serious deficiencies in glucose and oxygen supply to regions of brain tissue, thus promoting cellular damage and death. The first part of this study was designed to assess the therapeutic benefits of Tat-NTS peptide against cerebral ischemic injury using an OGD/R cell model. Our results showed that the Tat-NTS peptide significantly blocked the interaction of ANXA1 with importin $\beta$ and that it correspondingly decreased the nuclear translocation and p53 binding of ANXA1 in neurons. Our previous work showed that in the nucleus ANXA1 acts as a co-factor of p53 and upregulates its transcriptional activity, leading to the expression of the pro-apoptosis gene Bid [15]. Consistent with this, the transcriptional activity of p53 was shown in the current study to be significantly decreased after treatment of cells with Tat-NTS peptide. Furthermore, consistent with its effect on p53 activity, treatment with Tat-NTS peptide resulted in a profound reduction in Bid expression.

Many studies have shown that OGD/R injury can induce neuronal cell apoptosis. In this study, we found that TatNTS markedly inhibited neuronal apoptosis. It has been widely documented that apoptosis occurs through two major pathways involving extrinsic and intrinsic apoptotic signaling and that the Bid molecule connects the two apoptosis pathways [41]. Our study confirmed that treatment with Tat-NTS peptide decreased tBid, cleaved caspase-9, cleaved PARP, cleaved caspase-3 expression, and the cytosolic release of cytochrome $c$ from the mitochondria in neurons subjected to OGD/R. These results showed that Tat-NTS peptide could inhibit the activation of intrinsic apoptosis pathway by blocking the expression and activation of Bid. Furthermore, Tat-NTS peptide did not impair the viability or survival of hippocampal neurons under normal conditions. Collectively, these results show that the Tat-NTS peptide exerts a robust neuroprotective effect in ischemic brain by inhibiting neuronal apoptosis.

We further examined whether the Tat-NTS peptide was neuroprotective in a mouse focal cerebral ischemia model. It has been reported that thousands of neurons degenerate during the early stage of transient focal cerebral ischemic injury [42]. In this study, we found that injection of the TatNTS peptide resulted in efficient transfer of the peptide into brain tissue and that it produced therapeutic effects when 


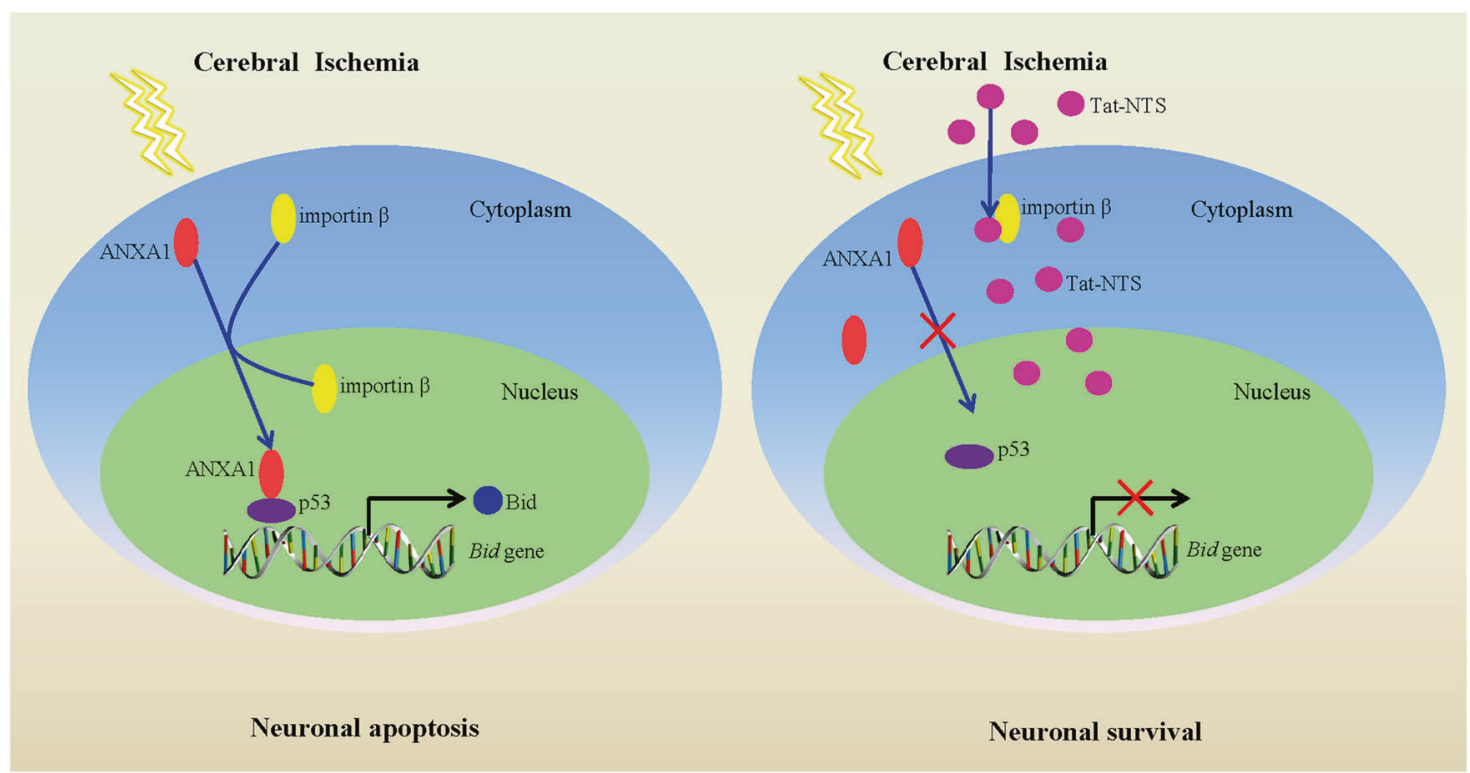

Fig. 8 Schematics summarize the major findings of the current study. The Tat-NTS peptide specifically blocked the interaction of ANXA1 with importin $\beta$ and inhibited the nuclear translocation of ANXA1.
Consequently, Tat-NTS peptide inhibited the transcriptional activity of p53, decreased Bid expression, and eventually improved the survival of neurons subjected to cerebral ischemia and reperfusion injury administered up to $6 \mathrm{~h}$ after stroke, whereas the therapeutic time window for $\mathrm{PAA}$ is $<4.5 \mathrm{~h}$ following the onset of stroke [43]. Nevertheless, more detailed studies will be needed to define the exact therapeutic window. Our results also showed that Tat-NTS significantly attenuated the number of TUNEL $^{+}$cells, suggesting that Tat-NTS rescues neurons that have undergone ischemic injury from degeneration and apoptosis. Furthermore, administration of the Tat-NTS peptide significantly attenuated the brain ischemic infarct volume, and decreased neurological deficit scores. Moreover, further work revealed that Tat-NTS peptide improves the learning, memory, and motor function of mice with cerebral ischemic injury. It would be therapeutically relevant to evaluate the neuroprotective effects of administration of Tat-NTS peptide by other peripheral administration routes such as subcutaneous, intravenous, and intraperitoneal injection. Future studies in our laboratory will address these issues.

In summary, this study verifies that the administration of Tat-NTS peptide specifically inhibits the nuclear translocation of ANXA1 by blocking the interaction of ANXA1 with importin $\beta$ in neuronal cells. Furthermore, the in vitro and in vivo data show that treatment of cells and animals with Tat-NTS peptide dramatically attenuates neuronal apoptosis, reduces infarct volume, and results in improved neurological functional outcome after focal cerebral ischemia (Fig. 8). Our data suggest that decreased neuronal apoptosis is one of the beneficial effects of treatment with Tat-NTS peptide after focal cerebral ischemic injury. Moreover, our data show that the Tat-NTS peptide has an extended efficacy with a remarkably long time window ( $>6$ h) for the treatment of stroke. Therefore, the fusion peptide Tat-NTS may represent a promising agent with potential clinical application in cerebral stroke and possibly in other types of neurodegenerative disease.

\section{Materials and methods}

\section{Animals}

Male C57BL/6 mice (14 weeks of age, weighing 22-25 g) and C57BL/6 mouse embryos (E18.5) were obtained from the Experimental Animal Center of Tongji Medical College, Huazhong University of Science and Technology. All animal experiments in this study were approved by the Ethics Committee for Animal Experimentation of Huazhong University of Science and Technology in strict accordance with the Guide for the Care and Use of Laboratory Animals published by the U.S. National Institutes of Health. Efforts were made to minimize animal suffering and to reduce the number of animals used.

\section{Reagents and antibodies}

The Tat-NTS $\left(\mathrm{NH}_{2}\right.$-YGRKKRRQRRR-RSFPHLRRVF$\left.\mathrm{CONH}_{2}\right)$ and Tat-Scr $\left(\mathrm{NH}_{2}\right.$-YGRKKRRQRRRFRLPSVHRFR-CONH ${ }_{2}$ ) peptides with $99 \%$ purity were synthesized by Moon Biosciences (Wuhan, China). Importazole was obtained from Calbiochem (401105; 
Darmstadt, Germany). The In Situ Cell Death Detection Kit was purchased from Sigma-Aldrich China (11684795910). The antibodies used in this study and the dilutions used were as follows: anti-ANXA1 (sc-12740, 1:500), anti-p53 (sc-126, 1:1000), anti-Bid (sc-11423, 1:1000), anti-p65(sc71675), anti- $\alpha$-tubulin (sc-69970, 1:2000), and anti- $\beta$-actin (sc-47778, 1:1000) were purchased from Santa Cruz Biotechnology (Dallas, Texas, USA); anti-importin $\beta$ (51186, 1:1000), anti- $\beta$-catenin (8480, 1:1000), anti-Histone H3 (4499, 1:2000), anti-cleaved caspase-9 (9505, 1:500), anticytochrome $c$ (12963, 1:1000), anti-cox IV (4850, 1:1000), anti-cleaved caspase-3 (9664, 1:1000), and anti-cleaved PARP (5625, 1:1000) were obtained from Cell Signaling Technology (Danvers, Massachusetts, USA); anti-tBid (ab10640, 1:1000) was purchased from Abcam (Cambridge, Massachusetts, USA); and anti-NeuN (MAB377, 1:250) was purchased from Millipore Biotechnology (Schwalbach, Germany). Alexa Fluor 594-conjugated AffiniPure Goat Anti-Rabbit IgG $(\mathrm{H}+\mathrm{L})$ and Alexa Fluor 488-conjugated AffiniPure Goat Anti-Mouse IgG $(\mathrm{H}+\mathrm{L})$ were obtained from Jackson Immuno Research (West Grove, Pennsylvania, USA). All other reagents are commercially available and were used as received.

\section{Transient focal cerebral ischemia}

Transient focal cerebral ischemia was produced by transient occlusion of the MCAO of mice using the intraluminal filament technique as previously described with minor modifications [44]. Briefly, mice were anesthetized with 5\% chloral hydrate $(350 \mathrm{mg} / \mathrm{kg}$, i.p. $)$; the rectal temperature of the anesthetized animals was maintained at $37 \pm 0.5^{\circ} \mathrm{C}$ using a homeothermic blanket (Harvard Apparatus, Holliston, Massachusetts, USA). The right common, external, and internal carotid arteries (ICAs) were exposed, and the common carotid arteries were ligated with sutures. A 7/0 silicone-coated monofilament nylon suture $(0.22-0.23 \mathrm{~mm}$ in diameter) with a rounded tip was gently introduced into the ICAs through the external carotid artery (ECA) stump and advanced $10 \pm 0.5 \mathrm{~mm}$ until the rounded tip reached the entrance to the right middle cerebral artery and a slight resistance was felt. Successful occlusion was validated by laser Doppler flowmetry (PeriFlux 5000, Järfälla, Sweden). One hour after the onset of occlusion, the nylon monofilament was withdrawn, and samples of brain tissue were collected at various time points thereafter.

\section{Administration of peptides}

Animals that had been subjected to 1-h MCAO were immediately injected with Tat-NTS or Tat-Scr peptide at a single dose of $0.5,1,2,5$, or $10 \mathrm{mg} / \mathrm{kg}$ body weight by unilateral i.c.v. administration in vehicle $(0.9 \%$ saline). For i.c.v. injection, the mice were anesthetized while on the ear bars of a stereotaxic apparatus (KOPF, Tujunga, California, USA), and the peptides were injected into the right lateral ventricle $(0.23 \mathrm{~mm}$ anteroposterior and $1.0 \mathrm{~mm}$ lateral to bregma; depth, $2.2 \mathrm{~mm}$ ) through a stepper-motorized microsyringe (Hamilton, Bonaduz, Switzerland) at a rate of $1 \mu \mathrm{l} / \mathrm{min}$. For the time window experiments, mice were injected with vehicle, Tat-NTS, or Tat-Scr peptide (2 $\mathrm{mg} / \mathrm{kg}$ body weight, i.c.v.) 3,6 , or $9 \mathrm{~h}$ after MCAO. The peptides were numbered, and the investigators were blinded with respect to which peptide was injected in all experiments.

\section{Primary hippocampal neuronal cultures}

Primary hippocampal neurons were cultured as previously described [15]. In brief, hippocampal neurons were isolated from C57BL/6 mouse embryos (E18.5) under a dissection microscope, washed with D-Hank's solution under sterile conditions and seeded at a density of $1 \times 10^{6}$ cells $/ \mathrm{cm}^{2}$ on plates coated with poly-L-lysine $(50 \mathrm{mg} / \mathrm{mL}$ ) (SigmaAldrich, St. Louis, Missouri, USA). The cells were grown in neurobasal medium supplemented with 2\% B27 (Gibco, Gaithersburg, Maryland, USA), $1 \%$ glutamine, and $1 \%$ penicillin/streptomycin (ThermoFisher Scientific, Waltham, Massachusetts, USA) at $37^{\circ} \mathrm{C}$ in a humidified incubator in air containing $5 \% \quad \mathrm{CO}_{2}$. The purity of the neurons was confirmed by NeuN immunofluorescence at 5 days after plating; $>95 \%$ of the cells in the culture were positive for NeuN (data not shown).

\section{Plasmids construction and transfection}

For Co-IP experiments (Figs. 1b, f), to generate GFP tagged full-length ANXA1 and truncated mutant constructs, specific primers were designed as described in Supplementary Table 1, and each truncated mutant was by amplified RTPCR, followed by cloning into the eukaryotic expression vector pEGFP-N1 vector (Clontech, Mountain View, California, USA). For fluorescence assay (Figs. 1c, d, g), to generate $\beta$-gal-GFP tagged ANXA1 truncated mutant constructs, each truncated mutant was by amplified RT-PCR, followed by cloning into the pEGFP-N1- $\beta$-gal vector. For the domain swapping experiments (Fig. 1h), oligonucleotides coding the NTS were cloned into the pEGFP-N1- $\beta$-gal vector. All mutations were verified by sequencing (performed by Sangon Biotech, Shanghai, China). HEK293 cells were cultured in Dulbecco's modified Eagle's medium (DMEM; Gibco) supplemented with 10\% fetal bovine serum using standard cell culture conditions. Confluent cell layers were split twice per week. Cell transfections were performed using Lipofectamine 2000 according to the manufacturer's instructions. 


\section{Oxygen-glucose deprivation and reperfusion}

We established an in vitro model of ischemic stroke by exposing cultured neurons to $\mathrm{OGD} / \mathrm{R}$ as previously described [15]. The neurons were gently rinsed with phosphate-buffered saline (PBS) three times, glucose-free DMEM that had been preheated to $37^{\circ} \mathrm{C}$ was added to the culture dish, and the dish was placed in an anaerobic incubator under a $95 \% \mathrm{~N}_{2}$ and $5 \% \mathrm{CO}_{2}$ atmosphere at $37{ }^{\circ} \mathrm{C}$ for $1 \mathrm{~h}$. The glucose-free DMEM was then replaced with normal neurobasal medium, and the cells were incubated under normoxic conditions in a humidified $5 \% \mathrm{CO}_{2}$ cell culture incubator for an additional $24 \mathrm{~h}$.

\section{Cell viability analysis and cell survival assays}

The CCK-8 assay was conducted to determine cell viability as previously described [45]. Briefly, neurons were seeded in 96-well plates at a density of $1 \times 10^{4}$ cells per well using standard neuronal culture conditions. After subjection of the cells to OGD/R, $10 \mu \mathrm{l}$ of CCK-8 was added to each well, the cells were incubated for an additional $4 \mathrm{~h}$, and the absorbance of each well at $450 \mathrm{~nm}$ was measured using a Benchmark Microplate Reader (ThermoFisher Scientific). For the survival assays, cells that had undergone the indicated treatments were fixed in $4 \%$ formaldehyde for $15 \mathrm{~min}$ and permeabilized with $0.1 \%$ Triton X-100. Thereafter, the neurons were incubated with $50 \mu \mathrm{l} /$ well TUNEL reaction mixture for $1 \mathrm{~h}$ and with $1 \mathrm{mg} / \mathrm{ml}$ of 2-(4-Amidinophenyl)6-indolecarbamidine dihydrochloride (DAPI) (SigmaAldrich) for $10 \mathrm{~min}$ at $37^{\circ} \mathrm{C}$. The cells were then examined and counted immediately under fluorescence microscopy (IX73, Olympus, Japan).

\section{Protein extraction and preparation}

For extraction of whole-cell lysates, primary cultured neurons or brain tissues were lysed in radioimmunoprecipitation assay (RIPA) lysis buffer (Beyotime Institute of Biotechnology, Haimen, China) supplemented with protease inhibitor cocktail (Roche, Basel, Switzerland). The NE-PER ${ }^{\mathrm{TM}}$ Nuclear and Cytoplasmic Extraction Kit (ThermoFisher Scientific) was used to prepare nuclear and cytoplasmic extracts according to the instructions provided by the manufacturer. The enhanced BCA protein assay kit (ThermoFisher Scientific) was used to measure the protein concentrations of the extracts.

\section{Western blotting and immunofluorescent staining}

Western blotting analysis was conducted as previously described [15]. In brief, equivalent amounts of total protein were subjected to 10 or $12 \%$ sodium dodecyl sulfate-polyacrylamide gel electrophoresis (SDS-PAGE) and transferred to polyvinylidene difluoride (PVDF) membranes (Millipore). For immunofluorescence staining, cultured neurons (fixed in $4 \%$ paraformaldehyde) or coronal sections were gently rinsed twice with PBS solution, permeabilized with $0.5 \%$ Triton X-100 for 60 min, and blocked with $10 \%$ donkey serum for an additional $60 \mathrm{~min}$. The neurons or sections were then incubated with antigenspecific primary antibodies [anti-ANXA1 (1:200) and antiNeuN (1:250)] overnight at $4{ }^{\circ} \mathrm{C}$. The samples were then rinsed thoroughly three times in $0.25 \%$ Tween-20 in PBS and incubated with secondary antibodies at room temperature for $60 \mathrm{~min}$. DAPI was used for counterstaining of the nuclei. The cells were examined and counted immediately under fluorescence microscopy (IX73, Olympus, Tokyo, Japan).

\section{Co-IP analysis}

Cell lysates or tissue homogenates (600 $\mu$ g of protein) were diluted fourfold with IP buffer (Beyotime Institute of Biotechnology) containing protease inhibitor cocktail. The samples were precleared for $1 \mathrm{~h}$ with $10 \mu \mathrm{l}$ of protein $\mathrm{A} / \mathrm{G}$ PLUS-Agarose beads (Santa Cruz Biotechnology), and then centrifuged to remove proteins that adhered nonspecifically to the protein $A / G$ beads. The supernatant was incubated with $2 \mu$ g of ANXA1 antibody or control IgG with gentle rotation overnight at $4{ }^{\circ} \mathrm{C}$. Thereafter, $20 \mu$ of protein $\mathrm{A} / \mathrm{G}$ PLUS-Agarose beads was added, and the samples were incubated at $4{ }^{\circ} \mathrm{C}$ for an additional $4 \mathrm{~h}$. The precipitates were washed three times with IP buffer, denatured in $30 \mu \mathrm{l}$ loading buffer, boiled at $95^{\circ} \mathrm{C}$ in a block heater (ThermoFisher Scientific) and subjected to SDS-PAGE.

\section{Luciferase reporter assay}

The luciferase reporter assay was performed according to the protocol provided with the Dual-Luciferase Reporter Assay System (Promega, Madison, Wisconsin, USA). Briefly, the pGL4.38 [luc2P/p53 RE/Hygro vector (Promega)] was transfected into neuronal cells. Forty-eight hours later, the cells were subjected to OGD/R; the cells were then harvested, and the firefly and renilla luciferase activities of the lysates were measured. The normalized values (renilla/firefly activity) were used for analysis. The experiments were performed in triplicate.

\section{Real-time quantitative PCR}

Total RNA was extracted using TRIzol reagent (Invitrogen, Carlsbad, California, USA), and $1 \mu \mathrm{g}$ RNA was reversetranscribed into complementary DNA (cDNA) using the ReverTra Ace- $\alpha$-TM First Strand cDNA Synthesis Kit 
(Toyobo, Osaka, Japan). Quantitative PCR was conducted using SYBR Green Premix Ex Taq (Takara, Tokyo, Japan) on an ABI StepOnePlus (Applied Biosystems, Waltham, Massachusetts, USA). The primers used were as follows: Bid, 5'-GCCTGTCGGAGGAAGACAAA-3' (forward) and 5'-GTGGAAGACATCACGGAGCA-3' (reverse); $\beta$-actin, $5^{\prime}$-GGCTGTATTCCCCTCCATCG-3' (forward) and 5'CCAGTTGGTAACAATGCCATGT-3' (reverse).

\section{Neurological score}

At 24,48 , and $72 \mathrm{~h}$ of reperfusion after MCAO, the neurological performance of the animals was assessed based on a mNSS that included motor tests (including flexion of the forelimb, flexion of the hindlimb, and head movement, scored on a scale of 0-6), beam balance tests (scored on a scale of 0-6), and tests for the presence of reflexes and abnormal movements (scored on a scale of 0-2). Cumulative scores of 1-4, 5-9, and 10-14 indicate mild, moderate, and severe injury, respectively. Neurological function was assessed by blinded independent research fellows.

\section{TTC staining}

To confirm the neuroprotective effects of Tat-NTS peptide, the animals' brains were removed rapidly and frozen immediately at $-80^{\circ} \mathrm{C}$ for $5 \mathrm{~min}$. The whole brain was then sectioned into 2-mm coronal slices using a brain matrix. The sections were stained with 2\% TTC (Sigma-Aldrich) in PBS at $37{ }^{\circ} \mathrm{C}$ for $20 \mathrm{~min}$ and fixed in $4 \%$ paraformaldehyde. The infarct volume was measured using Image-Pro Plus image analysis software as previously described.

\section{MWM test}

In this study, the spatial learning and memory of mice were assessed using the MWM test as described previously [46]. Briefly, the water maze consisted of a circular tub $(120 \mathrm{~cm}$ diameter, $60 \mathrm{~cm}$ high) filled with opaque water and a round platform $(6 \mathrm{~cm}$ diameter) that was submerged $1 \mathrm{~cm}$ beneath the surface of the water. The tub was located in an environment that was rich in visual cues external to the maze. Before the start of the trials, the animals were allowed to acclimate to the testing environment for $20 \mathrm{~min}$. Invisible platform training was conducted on 6 consecutive days, and each session consisted of four trials. For each trial, the animal was released from the wall of the tank and allowed to search for and stand on the hidden platform within the 60 -s trial period; if the animal did not reach the platform in the allotted time, it was manually guided to it. A probe test was conducted $24 \mathrm{~h}$ after the completion of training. In the test, the platform was removed, and the animal's performance was recorded for $60 \mathrm{~s}$. The latency of reaching the platform, the time spent in each quadrant, and the number of times the animal crossed the platform area were recorded.

\section{Open-field test}

An open-field test was used to evaluate the animals' locomotion after surgery or treatment. Mice were first placed in the center of an open-field apparatus $\left(50 \times 50 \times 30 \mathrm{~cm}^{3}\right)$ for $30 \mathrm{~min}$ and then habituated by placing them in the same chamber for 10 min per day on 3 consecutive days. After the 3-day habituation period, the animals' general locomotion was assessed for a 10-min period. Specifically, we measured distance moved in the arena. After removal of each animal, the open-field arena was cleaned.

\section{Statistical analysis}

All values are expressed as the mean \pm S.E.M. of at least three independent experiments. The data were analyzed using the statistical software GraphPad Prism (version 6.03, GraphPad Software, Inc). Differences between two groups were assessed using Student's unpaired $t$-test (two-tailed). The variance among multiple groups was determined by one-way or two-way ANOVA with/without repeated measures followed by the Newman-Keuls test. A value of $P<$ 0.05 was considered to indicate statistical significance.

Acknowledgements The current studies were supported by funding from the National Natural Science Foundation of China (no. 31771126, 31471015), China Postdoctoral Science Foundation (no. 2017M620310).

Author contributions JS, XL, and YZ conceived and designed the project; XL, LZ, and QX performed the experiments; LL, MM, and HJZ gave much valuable advice and conducted the data analysis; JS and XL wrote the manuscript with approval from all authors.

\section{Compliance with ethical standards}

Conflict of interest The authors declare that they have no conflict of interest.

\section{References}

1. Hankey GJ. Stroke. Lancet. 2017;389:641.

2. Benjamin EJ, Blaha MJ, Chiuve SE, Cushman M, Das SR, Deo R, et al. Heart disease and stroke statistics-2017 update: a report from the American Heart Association. Circulation. 2017;135: e146-603.

3. Khandelwal P, Yavagal DR, Sacco RL. Acute ischemic stroke intervention. J Am Coll Cardiol. 2016;67:2631-44.

4. Moskowitz MA, Lo EH, Iadecola C. The science of stroke: mechanisms in search of treatments. Neuron. 2010;67:181-98.

5. Fransen PS, Berkhemer OA, Lingsma HF, Beumer D, La VDB, Yoo AJ, et al. Time to reperfusion and treatment effect for acute ischemic stroke: a randomized clinical trial. JAMA Neurol. 2016;73:190. 
6. Jauch EC, Saver JL, Adams HP, Bruno A, Demaerschalk BM, Khatri P, et al. Guidelines for the early management of patients with acute ischemic stroke. Stroke. 2013;44:870-947.

7. Broughton BR, Reutens DC, Sobey CG. Apoptotic mechanisms after cerebral ischemia. Stroke. 2009;40:e331.

8. Luo Y, Yang X, Zhao S, Wei C, Yin Y, Liu T, et al. Hydrogen sulfide prevents $\mathrm{OGD} / \mathrm{R}$-induced apoptosis via improving mitochondrial dysfunction and suppressing an ROS-mediated caspase3 pathway in cortical neurons. Neurochem Int. 2013;63:826-31.

9. Solito E, Mcarthur S, Christian H, Gavins F, Buckingham JC, Gillies GE. Annexin A1 in the brain--undiscovered roles? Trends Pharmacol Sci. 2008;29:135.

10. Sugimoto MA, Vago JP, Teixeira MM, Sousa LP. Annexin A1 and the resolution of inflammation: modulation of neutrophil recruitment, apoptosis, and clearance. J Immunol Res. 2016;2016:8239258. (2016-1-13) 2016

11. Yin Z, Xing L, Gong J, Lu L, Chen L, Lu Z, et al. Annexin A1 nuclear translocation induces retinal ganglion cell apoptosis after ischemia-reperfusion injury through the p65/IL-1 $\beta$ pathway. Biochim Biophys Acta. 2017;1863:1350-8.

12. Arora S, Lim W, Bist P, Perumalsamy R, Lukman HM, Li F, et al. Influenza A virus enhances its propagation through the modulation of Annexin-A1 dependent endosomal trafficking and apoptosis. Cell Death Differ. 2016;23:1243.

13. Gavins FN. Are formyl peptide receptors novel targets for therapeutic intervention in ischaemia-reperfusion injury? Trends Pharmacol Sci. 2010;31:266-76.

14. Zhao Y, Wang J, Jiang H, Yu Z, Li X, Shi J. Following OGD/R, annexin 1 nuclear translocation and subsequent induction of apoptosis in neurons are assisted by myosin IIA in a TRPM7 kinase-dependent manner. Mol Neurobiol. 2015;51:729.

15. Li X, Zhao Y, Xia Q, Zheng L, Liu L, Zhao B, et al. Nuclear translocation of annexin 1 following oxygen-glucose deprivationl [ndash]|reperfusion induces apoptosis by regulating Bid expression via p53 binding. Cell Death Dis. 2016;7:e2356.

16. Soderholm JF, Bird SL, Kalab P, Sampathkumar Y, Hasegawa K, Uehara-Bingen $\mathrm{M}$, et al. Importazole, a small molecule inhibitor of the transport receptor importin- $\beta$. ACS Chem Biol. 2011; 6:700-8.

17. Song H, Kim W, Kim SH, Kim KT. VRK3-mediated nuclear localization of HSP70 prevents glutamate excitotoxicity-induced apoptosis and $\mathrm{A} \beta$ accumulation via enhancement of ERK phosphatase VHR activity. Sci Rep. 2016;6:38452.

18. Herhaus L, Perezoliva AB, Cozza G, Gourlay R, Weidlich S, Campbell DG, et al. Casein kinase 2 (CK2) phosphorylates the deubiquitylase OTUB1 at Ser16 to trigger its nuclear localization. Sci Signal. 2015;8:ra35.

19. Dowdy SF. Transducible TAT-HA fusogenic peptide enhances escape of TAT-fusion proteins after lipid raft macropinocytosis. Nat Med. 2004;10:310.

20. Guidotti G, Brambilla L, Rossi D. Cell-penetrating peptides: from basic research to clinics. Trends Pharmacol Sci. 2017;38:406-24.

21. Hill MD, Martin RH, Mikulis D, Wong JH, Silver FL, Terbrugge $\mathrm{KG}$, et al. Safety and efficacy of NA-1 in patients with iatrogenic stroke after endovascular aneurysm repair (ENACT): a phase 2, randomised, double-blind, placebo-controlled trial. Lancet Neurol. 2012;11:942-50.

22. El ZI, Touchard E, Berdugo M, Abadie C, Kowalczuk L, Deloche C, et al. Subconjunctival injection of XG-102, a c-Jun N-terminal kinase inhibitor peptide, in the treatment of endotoxin-induced uveitis in rats. J Ocul Pharmacol Ther. 2015;31:17-24.

23. Aghazadeh Y, Martinez-Arguelles DB, Fan J, Culty M, Papadopoulos $\mathrm{V}$. Induction of androgen formation in the male by a TATVDAC1 fusion peptide blocking 14-3-3varepsilon protein adaptor and mitochondrial VDAC1 interactions. Mol Ther. 2014;22: 1779-91.
24. Shin MJ, Kim DW, Jo HS, Cho SB, Park JH, Lee CH, et al. TatPRAS40 prevent hippocampal HT-22 cell death and oxidative stress induced animal brain ischemic insults. Free Radic Biol Med. 2016;97:250-62.

25. Boudhraa Z, Bouchon B, Viallard C, D'Incan M, Degoul F. Annexin A1 localization and its relevance to cancer. Clin Sci. 2016;130:205.

26. Rosengarth A, Gerke V, Luecke H. X-ray structure of full-length annexin 1 and implications for membrane aggregation. J Mol Biol. 2001;306:489-98.

27. Jeong SA, Kim K, Lee JH, Cha JS, Khadka P, Cho HS, et al. Aktmediated phosphorylation increases the binding affinity of hTERT for importin $\alpha$ to promote nuclear translocation. J Cell Sci. 2015;128:2287-301.

28. Shin HY, Reich NC. Dynamic trafficking of STAT5 depends on an unconventional nuclear localization signal. J Cell Sci. 2013;126(Pt 15):3333-43.

29. Soniat M, Chook YM. Nuclear localization signals for four distinct karyopherin-beta nuclear import systems. Biochem J. 2015;468:353-62.

30. Yan W, Li R, He J, Du J, Hou J. Importin beta1 mediates nuclear factor-kappaB signal transduction into the nuclei of myeloma cells and affects their proliferation and apoptosis. Cell Signal. 2015;27:851-9.

31. Yu H, Shi L, Qi G, Zhao S, Gao Y, Li Y. Gypenoside protects cardiomyocytes against ischemia-reperfusion injury via the inhibition of mitogen-activated protein kinase mediated nuclear factor kappa B pathway in vitro and in vivo. Front Pharmacol. 2016;7:148.

32. Lu T, Bao Z, Wang Y, Yang L, Lu B, Yan K, et al. Karyopherinbeta1 regulates proliferation of human glioma cells via Wnt/ beta-catenin pathway. Biochem Biophys Res Commun. 2016; 478:1189-97.

33. Zhang J, Zhang J, Qi C, Yang P, Chen X, Liu Y. Activation of Wnt3alpha/beta-catenin signal pathway attenuates apoptosis of the cerebral microvascular endothelial cells induced by oxygenglucose deprivation. Biochem Biophys Res Commun. 2017;490: 71-77.

34. Kaufmann T, Strasser A, Jost PJ. Fas death receptor signalling: roles of Bid and XIAP. Cell Death Differ. 2012;19:42-50.

35. Culmsee C, Krieglstein J. Ischaemic brain damage after stroke: new insights into efficient therapeutic strategies. International Symposium on Neurodegeneration and Neuroprotection. EMBO Rep. 2007;8:129-33.

36. Bolhassani A, Jafarzade BS, Mardani G. In vitro and in vivo delivery of therapeutic proteins using cell penetrating peptides. Peptides. 2017;87:50-63.

37. Zhou HH, Tang Y, Zhang XY, Luo CX, Gao LY, Wu HY, et al. Delayed administration of Tat-HA-NR2B9c promotes recovery after stroke in rats. Stroke. 2015;46:1352-8.

38. Teves LM, Cui H, Tymianski M. Efficacy of the PSD95 inhibitor Tat-NR2B9c in mice requires dose translation between species. J Cereb Blood Flow Metab. 2015;36:0271678X15612099.

39. Cook DJ, Teves L, Tymianski M. A translational paradigm for the preclinical evaluation of the stroke neuroprotectant Tat-NR2B9c in gyrencephalic nonhuman primates. Sci Transl Med. 2012;4:154ra133.

40. Brooks H, Lebleu B, Vivès E. Tat peptide-mediated cellular delivery: back to basics. Adv Drug Deliv Rev. 2005;57: 559-77.

41. Rossi D, Gaidano G. Messengers of cell death: apoptotic signaling in health and disease. Haematologica. 2003;88:212-8.

42. Ansari S, Azari H, Mcconnell DJ, Afzal A, Mocco J. Intraluminal middle cerebral artery occlusion (MCAO) model for ischemic stroke with laser doppler flowmetry guidance in mice. J Vis Exp. 2011;51:e2879-e2879. 
43. Hacke W, Kaste M, Bluhmki E, Brozman M, Dávalos A, Guidetti $\mathrm{D}$, et al. Thrombolysis with alteplase 3 to $4.5 \mathrm{~h}$ after acute ischemic stroke. New Engl J Med. 2008;359:1317-29.

44. Xie XQ, Zhang P, Tian B, Chen XQ. Downregulation of NADdependent deacetylase SIRT2 protects mouse brain against ischemic stroke. Mol Neurobiol. 2017;54:7251-61.
45. Fan X, Zhao F, Wang X, Wu G. Doxorubicin-triggered selfassembly of native amphiphilic peptides into spherical nanoparticles. Oncotarget. 2016;7:58445-58.

46. Lei $\mathrm{P}$, Shan W, Jin $\mathrm{H}, \mathrm{Bi}$ L, Na W, Yan $\mathrm{H}$, et al. A novel mechanism of spine damages in stroke via DAPK1 and Tau. Cereb Cortex. 2015;25:4559-71. 